Ann. Biol. anim. Bioch. Biophys., I968, 8 (3), 445-447.

\title{
ABSORPTION DES MATIÈRES GRASSES PAR LA VOIE LYMPHATIQUE GHEZ LE VEAU PRÉRUMINANT
}

\author{
R. TOULLEC \\ Station de Recherches sur l'Élevage des Ruminants, \\ Centre de Recherches zootechniques et vétérinaires sur les Ruminants, \\ 63 - Theix près Clermont-Ferrand \\ Institut national de la Recherche agronomique
}

Trois veaux mâles F.F.P.N. âgés respectivement de 3,4 et 6 semaines ont été munis de canules du canal thoracique avec retour à la veine jugulaire interne, au niveau de la première côte gauche selon la technique de JoEL et SAUTTER (1963). Ces dérivations n'ont jamais pu être maintenues fonctionnelles pendant plus de 8 jours consécutifs par suite de la coagulation dans la canule de retour. Les mesures commençaient le $2^{\mathrm{e}}$ ou le $3^{\mathrm{e}}$ jour après l'opération. Les veaux ont reçu chaque jour une matière grasse différente : suif, saindoux, huiles d'arachide, de coprah et de palme, dans l'ordre indiqué dans le tableau I. Ces matières grasses étaient incluses sous forme homogénéisée dans des aliments d'allaitement à raison de $20 \mathrm{p}$. roo de la matière sèche. Chaque jour, les animaux ont reçu le matin 75 à roo $\mathrm{g}$ de la matière grasse à étudier et le soir un repas, pratiquement lipidoprive, de lait écrémé et d'amidon de maīs cru. Des prélèvements de lymphe, d'une durée de io à $20 \mathrm{mn}$, selon le débit, ont eu lieu par intervalles croissants au cours des 24 heures suivant l'ingestion de chaque matière grasse.

Les débits de lymphe ont été considérables, en moyenne de l'ordre de $45^{\circ} \mathrm{ml} / \mathrm{heure}$, ce qui s'accorde avec les résultats obtenus par SHANnon et LASCELLES (1967) sur des veaux recevant du lait entier. La quantité de lipides transportée par minute passait par un minimum de $20 \mathrm{mg}$ à jeun jusqu'à un maximum de I0o à $200 \mathrm{mg}$ (suivant les matières grasses ingérées et les animaux) 4 à I6 heures après le repas, pour décroître ensuite très rapidement. L'absorption paraissait pratiquement achevée 13 à 18 heures après le repas.

La composition en acides gras des lipides de la lymphe est très rapidement influencée par celle des matières grasses alimentaires pour devenir très proche de celle-ci au bout de 3 à 4 heures après le repas et le rester pendant 8 à I 2 heures environ, ce qui avait été observé chez plusieurs espèces monogastriques (cf. revue de Clement, I964).

Il n'y a de différences importantes de composition en acides gras entre les lipides de la lymphe et les matières grasses alimentaires qu'à la suite de l'ingestion d'acides gras courts et moyens (huile de coprah) : en supposant que le $\mathbf{C}_{14}$ est transporté en totalité par la voie lymphatique et qu'il n'y a pas d'apport endogène important de cet acide, on peut calculer que seulement des traces du $\mathrm{C}_{8}$, 36 p. I00 du $\mathrm{C}_{10}$ et 86 p. I00 du $\mathrm{C}_{12}$ ont emprunté la même voie. Cela s'accorde avec les résultats obtenus notamment par BLOOM, ChaIKoFF et ReINHARDT (I95I). L'apport endogène n'a d'influence notable 
R. TOULIEC

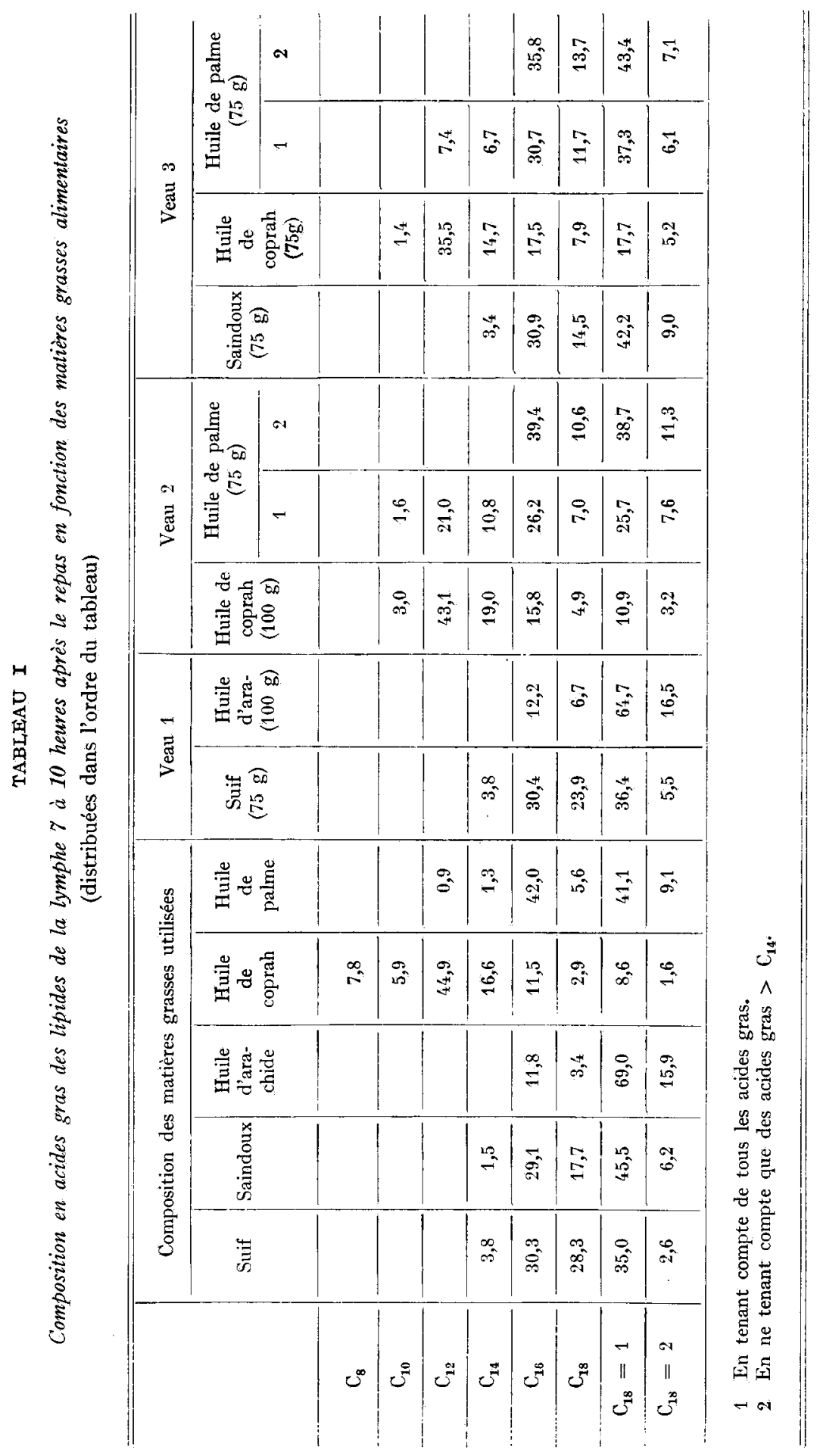


que sur les teneurs en acides gras peu abondants dans l'alimentation $\left(\mathrm{C}_{18}=2\right.$ du suif, $\mathrm{C}_{18}$ des huiles d'arachide, de coprah et de palme,), ce qui confirme les résultats de SAvary et Constantin (ig67).

Les matières grasses ingérées la veille n'ont pas d'incidence sur la composition en acides gras des lipides de la lymphe, sauf l'huile de coprah dont l'influence dure environ 40 heures. C'est ainsi qu'à la suite de l'ingestion de l'huile de palme (pratiquement dépourvue d'acides gras $<\mathrm{C}_{16}$ ) offerte 24 heures après celle de coprah, les lipides de la lymphe restent très riches en acides gras courts et moyens pendant encore au moins 16 heures. D'ailleurs en ne tenant compte que des acides gras $>\mathrm{C}_{14}$, on retrouve une composition en acides gras très proche de celle de l'huile de palme. Cette présence prolongée des acides gras courts et moyens peut être due à leur absorption plus lente (ce qui est peu probable), à leur stockage dans la paroi intestinale, à leur retour dans la lumière intestinale par l'intermédiaire de la bile ou à leur maintien sous forme circulante dans le plasma tant qu'ils n'ont pas été utilisés à des fins énergétiques ou à la synthèse d'acides gras longs.

Les lipides de la lymphe comprennent (moyenne de ro échantillons) : $75 \mathrm{p}$. Io0 de glycérides (essentiellement triglycérides), I8 p. roo de phospholipides, $5 \mathrm{p}$. Ioo d'esters du cholestérol et 2 p. 100 d'acides gras libres. Les triglycérides ont la composition en acides gras la plus proche de celle des matières grasses alimentaires. Les phospholipides sont beaucoup plus riches en acides stéarique et linoléique tandis que les esters du cholestérol contiennent moins d'acide stéarique et davantage d'acide linoléique. Les phospholipides et les esters du cholestérol comprennent peu d'acides gras courts.

En conclusion, chez le veau préruminant, les acides gras longs alimentaires empruntent surtout la voie lymphatique et contrairement à ce qui se produit chez le ruminant adulte (Lough, FelinskI et GARTON, I963), leur insaturation n'est pas modifiée au cours de la digestion et de l'absorption. Les acides gras $<\mathrm{C}_{16}$ passent d'autant moins dans la lymphe qu'ils sont plus courts. Le veau préruminant se comporte donc à cet égard en monogastrique fonctionnel.

Reģu pour publication en mai 1968.

\section{SUMMARY}

\section{LYMPH ABSORPTION OF LIPIDS IN THE MILK-FED CALF}

The variations in the composition of lymph lipids due to different dietary fats were studied in the milk-fed calf. Long-chained fatty acids are mainly transported through the lymphatic pathway and their unsaturated state is not altered throughout the digestive and absorptive process. $<\mathrm{C}_{18}$ fatty acids use the lymphatic pathway the less as they are shorter-chained. The milkfed calf thus behaves like a functional unigastric animal.

\section{RÉFÉRENCES BIBLIOGRAPHIQUES}

Bloom B., Chaikoff I. L., Reinhardt W. O., r95I. Intestinal lymph as pathway for transport of absorbed fatty acids of different chain lengths. Am. J. Physiol, 166, 451-455.

Clement G., I964. La digestion et l'absorption des graisses. J. Physiol., 56, IIr-I92.

Joel D. D., SautTer J. H., I96. Preparation of a chronic thoracic duct-venous shunt in calves. Proc. Soc. Exp. Biol. Med., 112, 856-859.

Lough A. K., Felinski I., Garton G. A., I963. Thoracic duct lymph lipids of the sheep. In Biochemical problems of lipids. Edited by Frazer A. C. Elsevier Publishing Company. Amsterdam-London-New York, p. 264-27x.

Savary A. D., Constantin M. J., 1967. Some experimental data on the endogenous chains in the triglycerides of rat thoracic duct lymph. Biochim. Biophys. Acta, 144, 549-555.

Shannon A. D., Lascelles A. K., 1967. A study of lipid absorption in young milk-fed calves with the use of a lymphatico-venous shunt for the collection of thoracic duct lymph. Aust. J. Biol. Sci., 20, 669-681. 